\title{
Predlicting Trait Competitive Anxiety, Competitive Aggression, and Competitive Anger based on Spiritual Intelligence in Veterans and Disabled Athletes
}

\section{ART ICLE INF O}

\section{Article Type}

Descriptive Study

\section{Authors}

Khajavi D.*PhD,

MirAli H. ${ }^{1} M S C$

How to cite this article
Khajavi D, MirAli H. Predicting
Trait Competitive Anxiety, Com-
petitive Aggression, and Com-
petitive Anger based on Spiritual
Intelligence in Veterans and
Disabled Athletes. Iranian Jour-
nal of War \& Public Health.
2017;9(3):163-168.

*Motor Behavior \& Sport Psychology Department, Sport Sciences Faculty, Arak University, Arak, Iran ${ }^{1}$ Motor Behavior \& Sport Psychology Department, Sport Sciences Faculty, Arak University, Arak, Iran

\section{Correspondence}

Address: Motor Behavior \& Sport Psychology Department, Sport Sciences Faculty, Arak University, Beheshty Street, Arak, Iran Phone: -

Fax: -

d-khajavi@araku.ac.ir

\section{Article History}

Received: May 29, 2016

Accepted: November 7, 2016

ePublished: July 27, 2017

\section{A B S T R A C T}

Aims In recent years, spirituality has been considered by health professionals as one of the existential dimensions of human beings due to their mental health effects. Spiritual intelligence is also an important factor affecting mental disorders such as anxiety, aggression and anger. Therefore, the purpose of this study was to predict competitive trait anxiety, competitive aggression and competitive anger based on the spiritual intelligence of veterans and disabled athletes.

Instrument \& Methods In this descriptive-correlational study in 2016, 80 veteran and disabled athletes from the Arak City, Iran, were selected through available sampling and participated in the study. Demographic characteristics questionnaire, spiritual intelligence questionnaire, competitive anger and aggression inventory and competitive trait anxiety questionnaire. To analyze the collected data, descriptive (mean and percent) and regression statistical tests were used.

Findings There was a significant negative relationship between spiritual intelligence and competitive anxiety $(\mathrm{r}=-0.22 ; \mathrm{p}<0.05)$, and spiritual intelligence and competitive anger and aggression $(\mathrm{r}=-0.27 ; \mathrm{p}<0.05)$. Spiritual intelligence could predict $4.9 \%$ of the variance of competitive anxiety $(\mathrm{p}=0.048)$ and $7.0 \%$ of the variance of competitive anger and aggression $(\mathrm{p}=0.017)$.

Conclusion Spiritual intelligence can reduce competitive anger and anger and competitive aggression in athletes with disabilities and veterans.

Keywords Spiritual Intelligence; Anxiety; Aggression; Anger; Veterans; Athletes

\section{CIT A T I O N LINKS}

[1] Mediating role of self-concept in perfectionism and mental ... [2] Assessment of mental health status ... [3] A Comparison of aggression, anxiety, and depression [4] Nursing concepts for health ... [5] Is spirituality intelligence? motivation cognition ... [6] Spiritual intelligence of nurses in two Chinese social systems ... [7] Developing a plan for pediatric spiritual ... [8] Relationship between anxiety and depression and ... [9] Personality and Health: The mediating role of trait [10] Religious coping and physiological ... [11] The mediating role of God attachment between religiosity ... [12] Communion and complaint: Attachment ... [13] Medicalsurgical nursing: Management for positive ... [14] Motor control and learning: A behavioral ... [15] Social psychology: Theories... [16] The aggression ... [17] On the relationship between mental ... [18] Comparison of spiritual intelligence ... [19] A comparison between intellectual aptitude and mental health in mothers having deaf, blind and ... [20] Relationship between religious / spiritual components, mental health and hope for the future in hospital staff of ... [21] Relationship between spiritual ... [22] The role of spiritual intelligence in perceived stress, anxiety ... [23] The relationship between spiritual intelligence and aggression among ... [24] Province comparison of general health between ... [25] The relationship between spiritual health and social anxiety ... [26] Study of relationship between spiritual health, anxiety ... [27] The study of relationship between spiritual intelligence ... [28] The development of a short scale measuring aggressiveness ... [29] Determination of validity and reliability of Farsi version ... [30] The relation between sports ... [31] Relationship of spirituality and forgiveness with family ... [32] Effect of training spiritual intelligence on quality of life ... [33] Relationship between spiritual health and quality ... [34] Relationship between spiritual health with quality ... [35] Role of sexual satisfaction, spiritual health, psychological ... [36] A model for controlling aggressiveness ... 


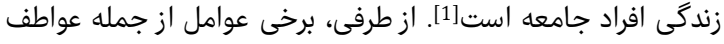

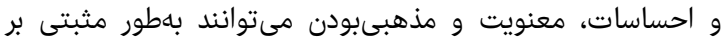

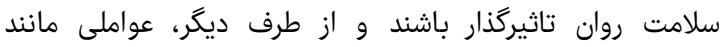

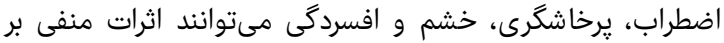

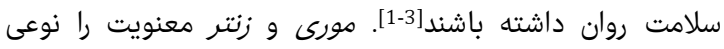

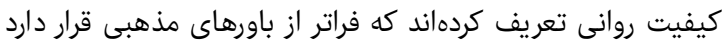

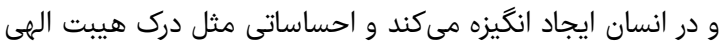

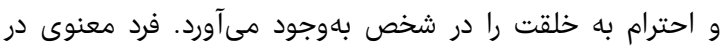

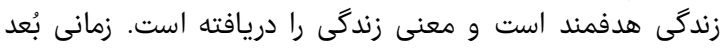

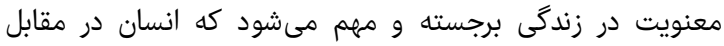

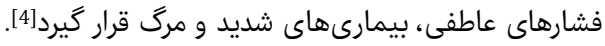

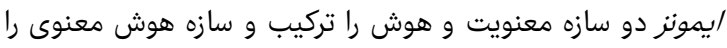

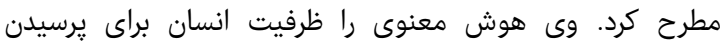

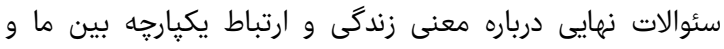

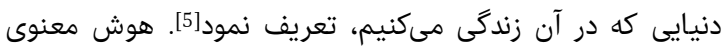

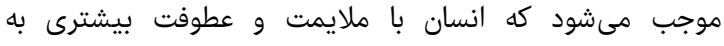

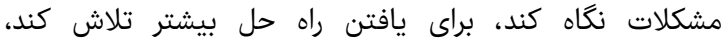

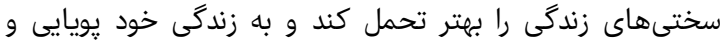

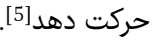

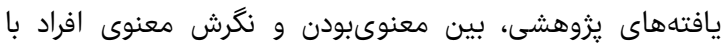

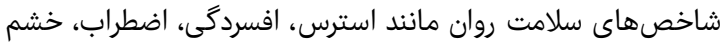

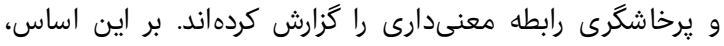

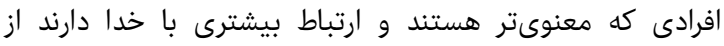

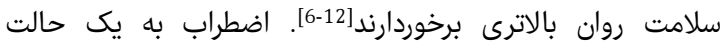

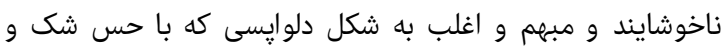

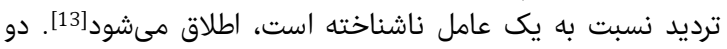

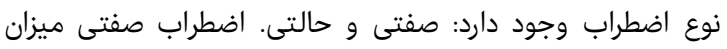

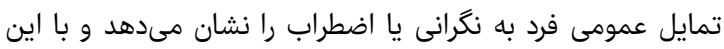

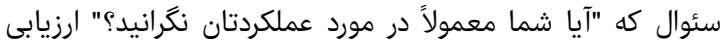

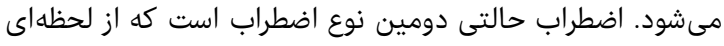

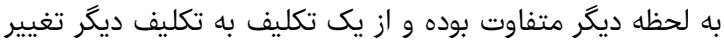

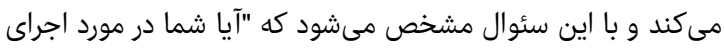

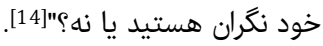
يكى از انواع اضطرابهاى صفتى، اضطراب صفاب صفتى ورزشى است كه إنى

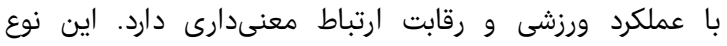

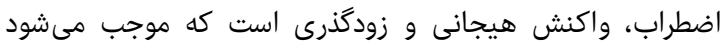

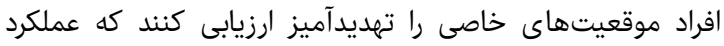

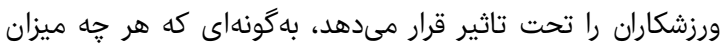

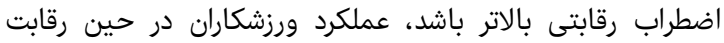

$$
\text { يإيينتر خواهد بود [14]. }
$$

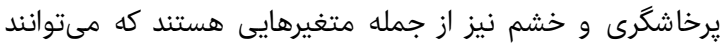

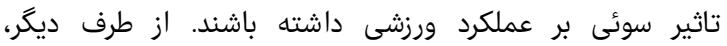

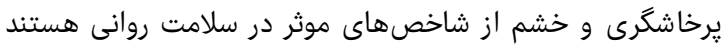

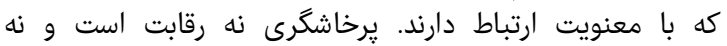

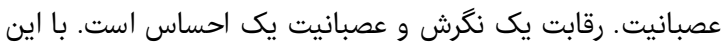

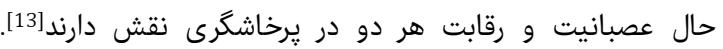

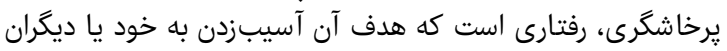

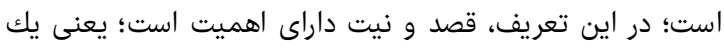

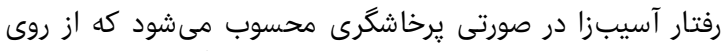

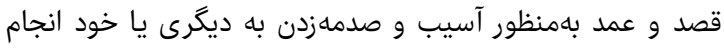

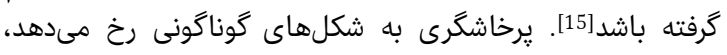

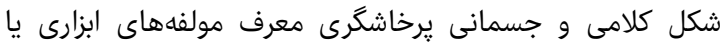

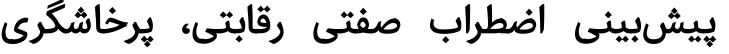 رقابتى و خشم رقابتى بر اساس هوش رابنى معنوى ورزشكاران جانباز و معلول وقابتي}

\author{
PhD داريوش خواجوى

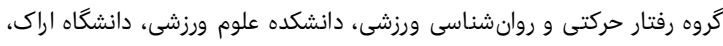

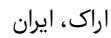

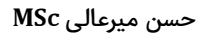 \\ كَره رفتار حركتى و روانش شناسى ورزشى، دانشكده علوم ورزشى، دانشكاه اراك،

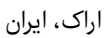

קكيده

اهداف: در سالهاى اخير، معنويت بهاعنوان يكى از ابعاد وجودى انسان،

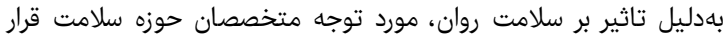

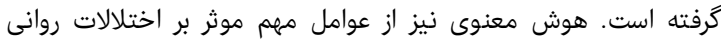

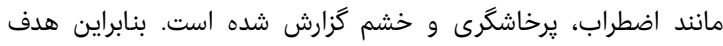

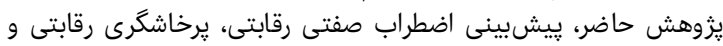

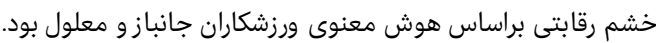

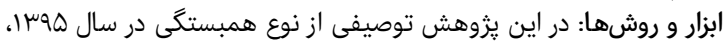

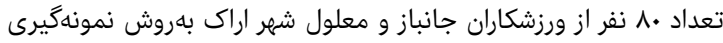

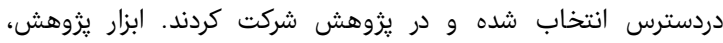

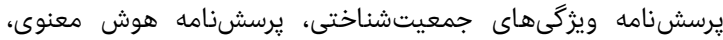

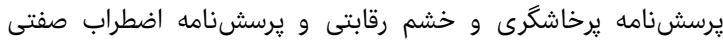

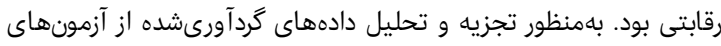

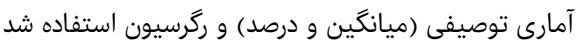

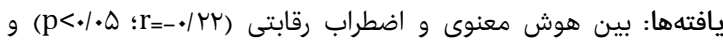

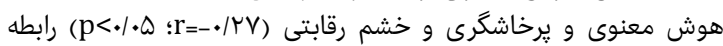

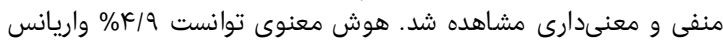

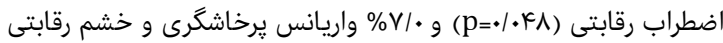
(p=•/・V)

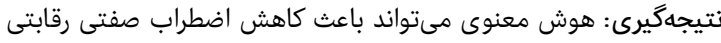

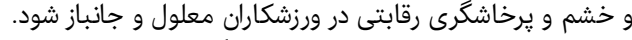
كليدواثهها: هوش معنوى، اضطراب رقابتى، يرخاشكرى، خشم، ورزشكاران وران جانباز

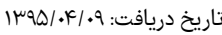

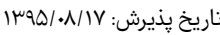
d-khajavi@araku.ac.ir :نويسنده مسئول:

مقدمه - - مقان

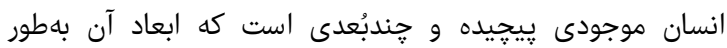

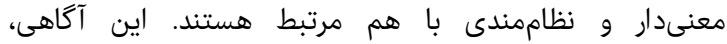

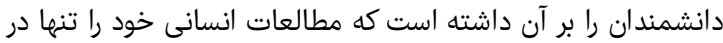

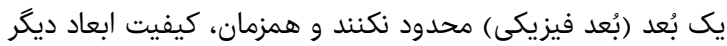

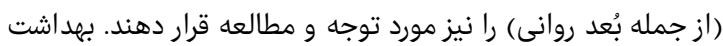

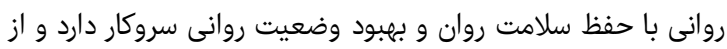

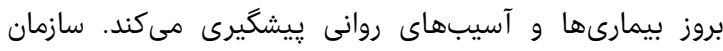

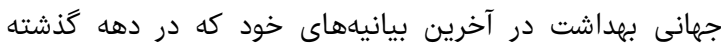

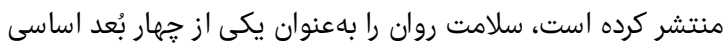

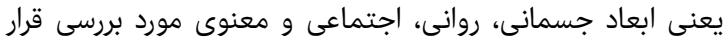

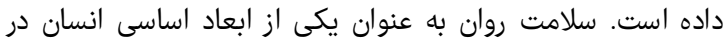

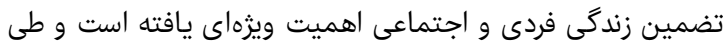

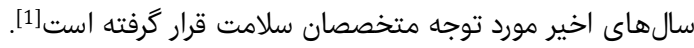

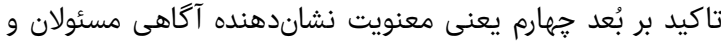

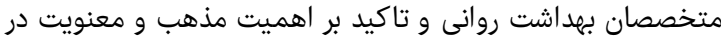

فصل نامه علمى - يزووهشى طب جانباز 


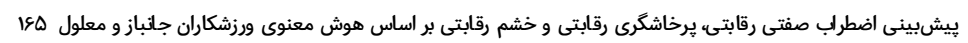

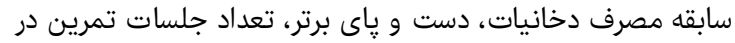

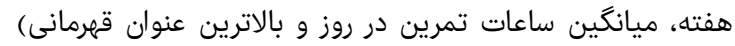

بود.

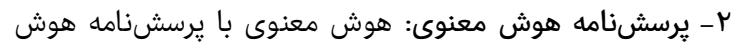

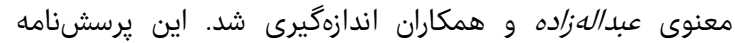

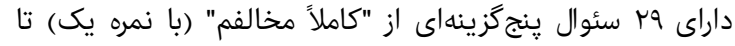

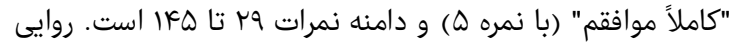

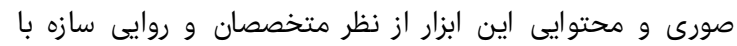

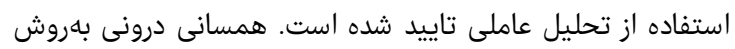

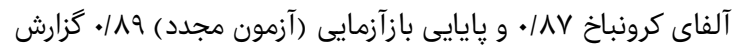

شدهاست[ن] (27].

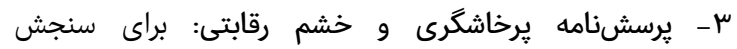

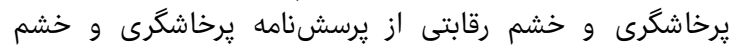

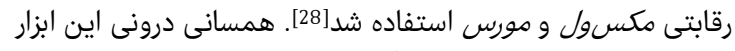

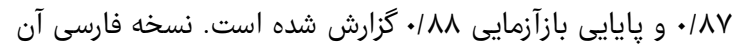

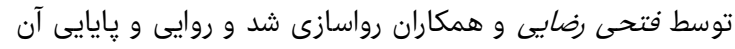

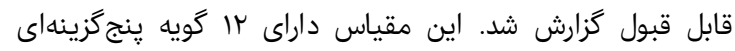

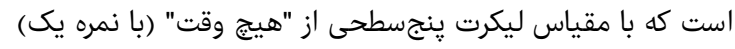

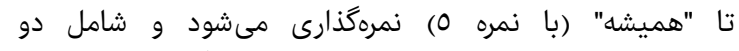

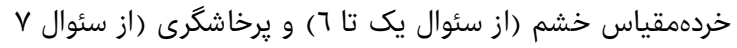

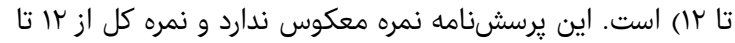

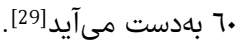

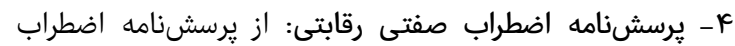

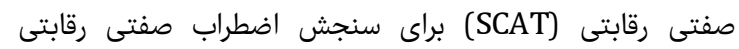

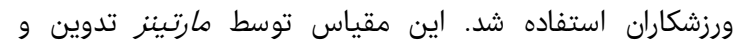

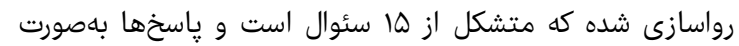

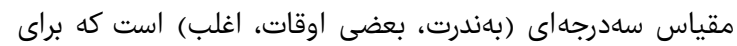

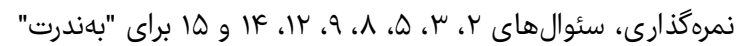

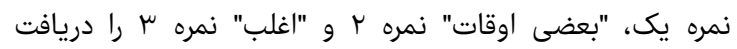

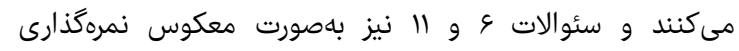

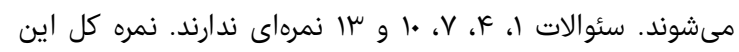

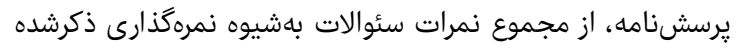

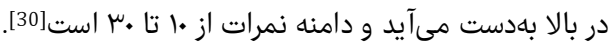

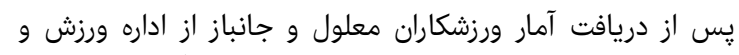

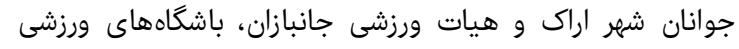

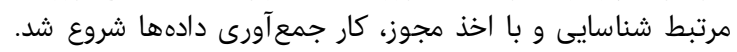

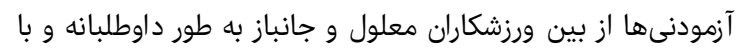

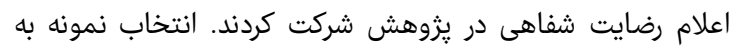

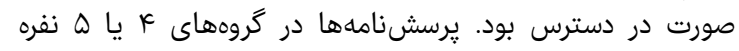

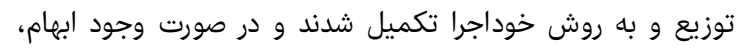

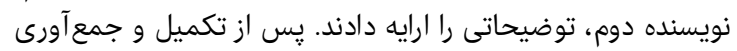

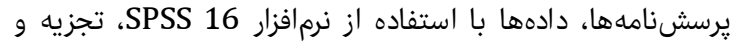
تحليل شدند. بهمنظور تجزيه و تحليل دادههاى گردآورىشده، از آمار توصيفى آدئى

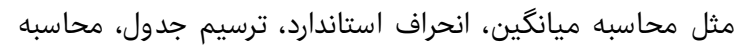

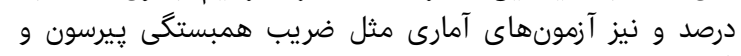
ركرسيون استفاده شد.

يافتهها

از بين آزمودنىهاى شركت شننده در يزوهش

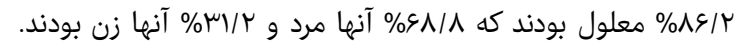

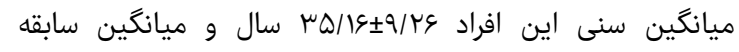

Iranian Journal of War and Public Health
رفتارى، خشم معرف جنبه هيجانى و خصومت معرف جنبه

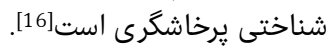

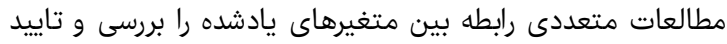

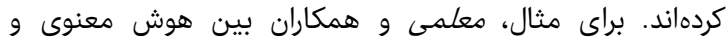

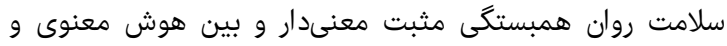

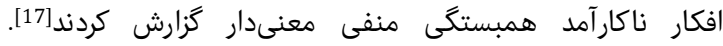

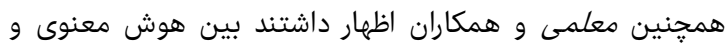

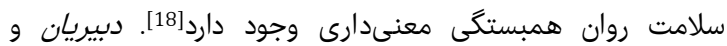

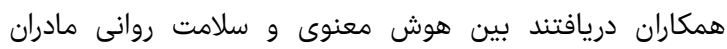

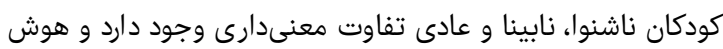

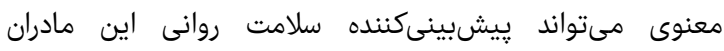

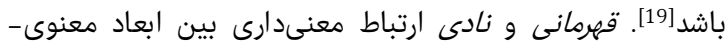

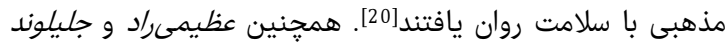

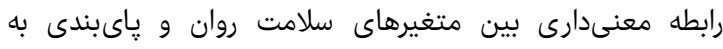

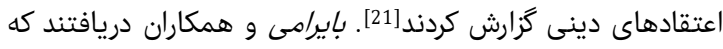

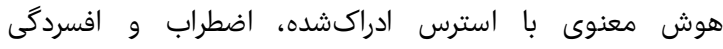

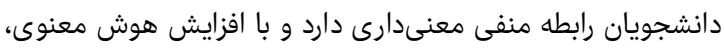

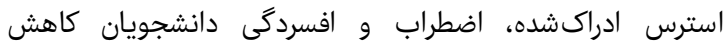

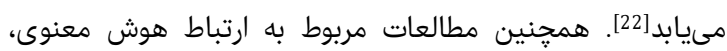

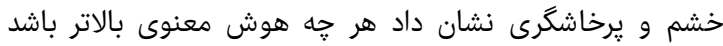

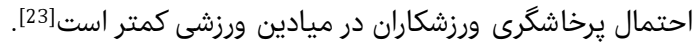

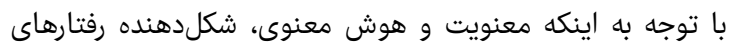

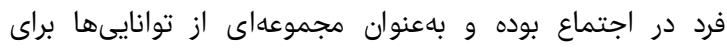

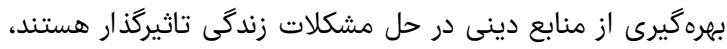

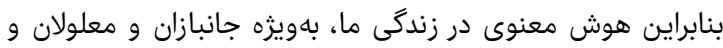

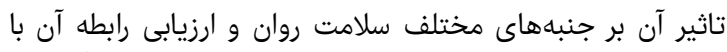

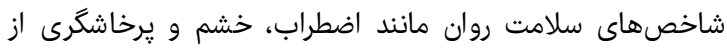

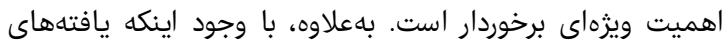

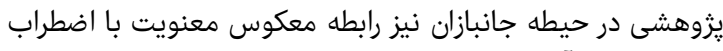

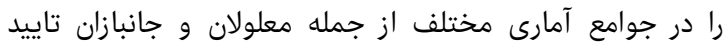

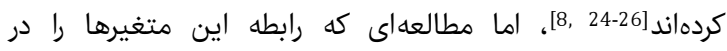

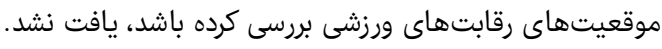

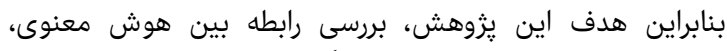

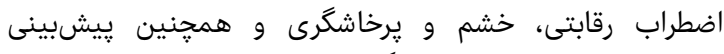

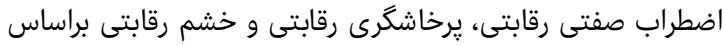

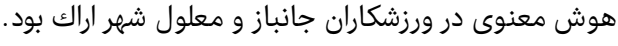

ابزار و روشها

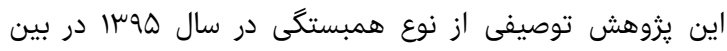

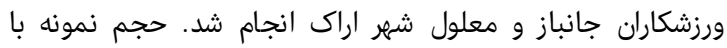

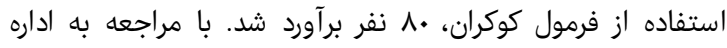

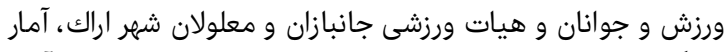

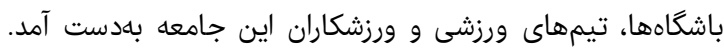

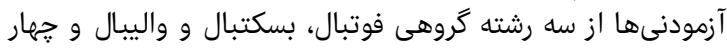

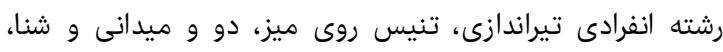

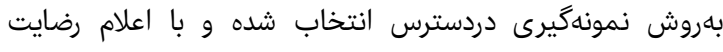

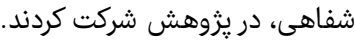

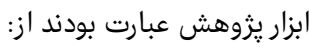

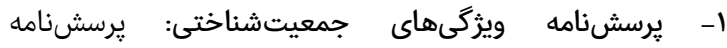

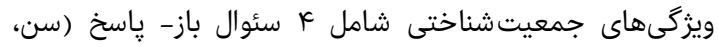

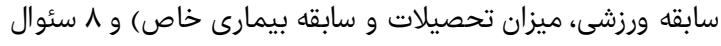

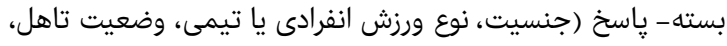




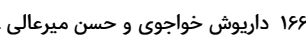

معنىدارى مشاهده شد، اما بين نمره هوش معنوى (نمره كل) و

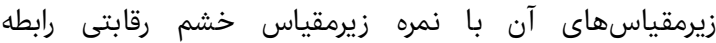

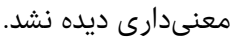

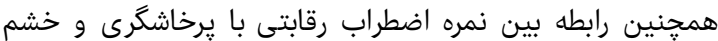

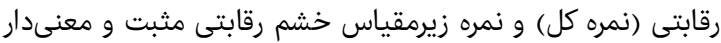

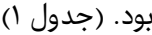

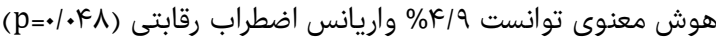

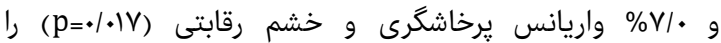
ييشبينى نمايد.

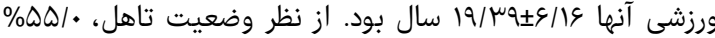

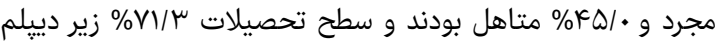

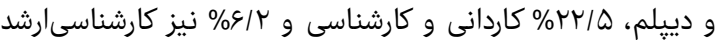

ميانگين نمرات هوش معنوى (نمره كل) و زيرمقياس زندگى

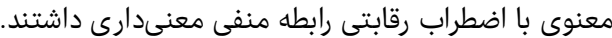

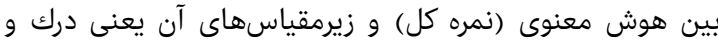

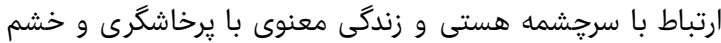

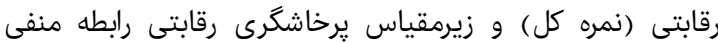

\begin{tabular}{|c|c|c|c|c|c|c|c|}
\hline 8 & $\omega$ & F & $\mu$ & $r$ & 1 & ميانگين & متغيرهاى يزوهش \\
\hline & & & & & 1 & $r M / V Y \pm \Delta / S V$ & 1- اضطراب ييشرقابتى \\
\hline & & & - & 1 & $\cdot / \mu r=*$ & $\vee \vee / V q \pm 1 \cdot / 9 ६$ & r - يرخاشگرى و خشم رقابتى (نمره كل) \\
\hline & & & 1 & $\cdot / \Lambda k^{* *}$ &.$/ 19$ & $r \Delta / \varepsilon H \pm \varepsilon / \Lambda F$ & " - زيرمقياس يرخاشگرى رقابتى \\
\hline & & 1 & $.10 \varphi^{\circ \circ}$ &.$/ 9 r^{* *}$ & $\cdot / \mu c^{* *}$ & $r r / l Y \pm r / l r$ & F - زيرمقياس خشم رقابتى \\
\hline & 1 & $-\cdot / 11$ & $-\cdot /\left.\right|^{w *}$ & $-\cdot / r V^{*}$ & $-\cdot / r r^{*}$ & $\| r \Lambda /|F \pm| Q / 19$ & ه- هوش معنوى (نمره كل) \\
\hline 1 &.$/ 91^{* *}$ & $-\cdot / 14$ & $-\cdot / \mu \mu^{*}$ & $-\cdot / Y K^{*}$ & $-\cdot / 10$ & $\Delta r / K G \pm \Delta / V Y$ & צ- زيرمقياس درك و ارتباط با سرجشمه هستى \\
\hline.$/ V * *$ & $.19 \mu=0$ & $-\cdot / r$ &.$- / r \Delta^{\circ}$ & $-\cdot / r \Delta^{*}$ &.$- / 4 \varphi^{*}$ & $V \bowtie / \& \wedge \pm q / \& V$ & V- زيرمقياس زندگى معنوى \\
\hline
\end{tabular}

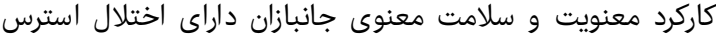

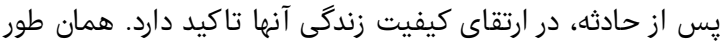

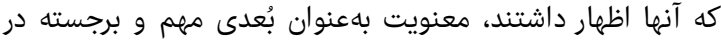

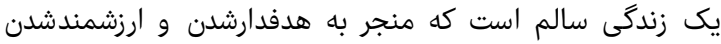

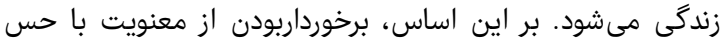

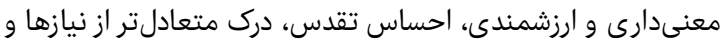

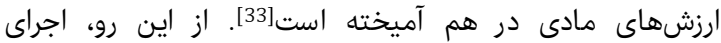

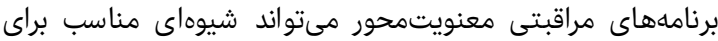
كاهش اضطراب باشد [26].

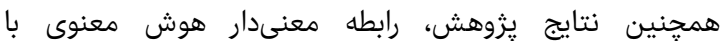

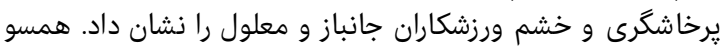

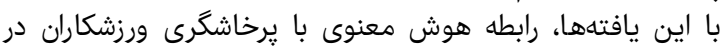

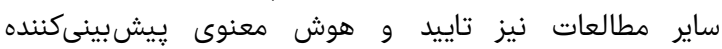

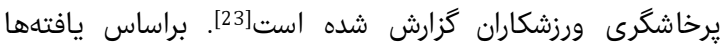

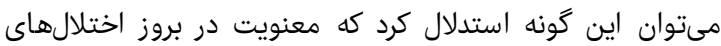

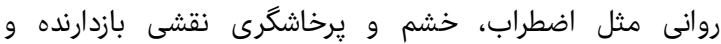

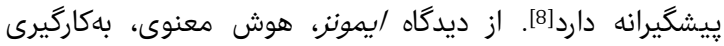

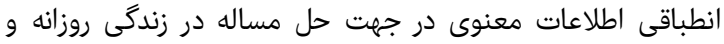

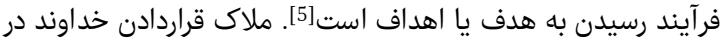

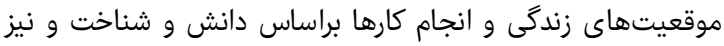

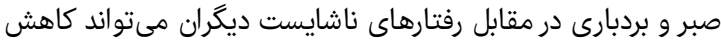

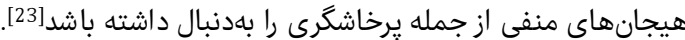

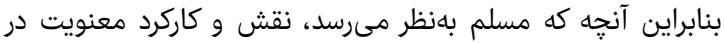

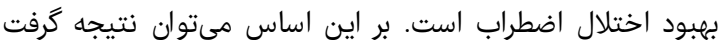

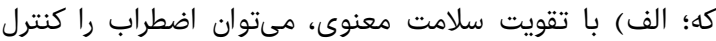

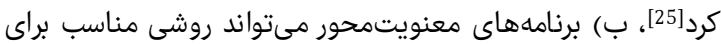

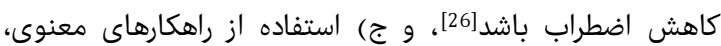

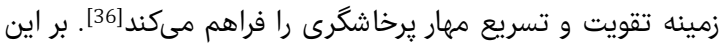

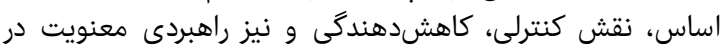

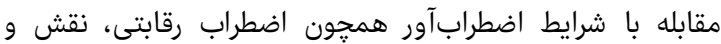

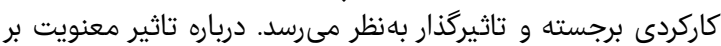

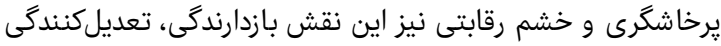

بحث

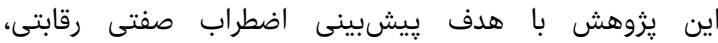

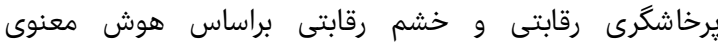

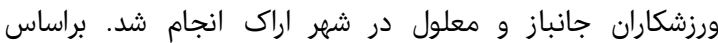

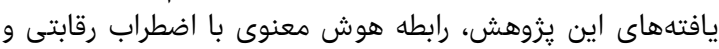

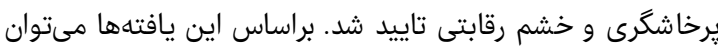

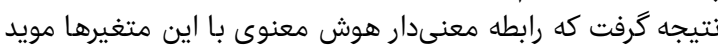

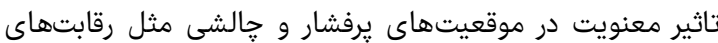

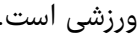
يافتههاى اين يزوهش نشان دان داد كه معنويت از يك طرف مى دواند

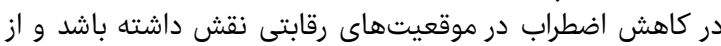

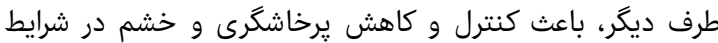

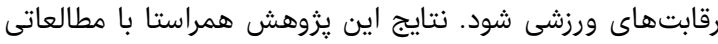

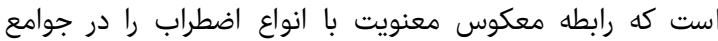

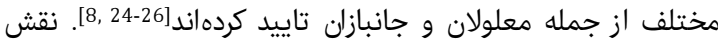

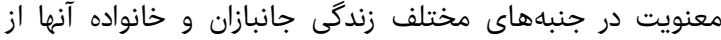

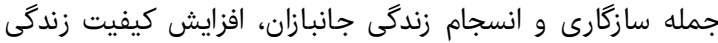

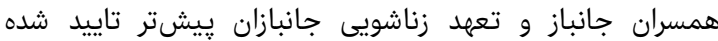

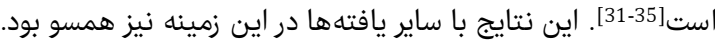

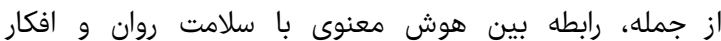

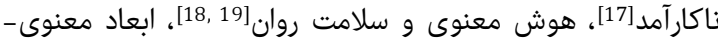

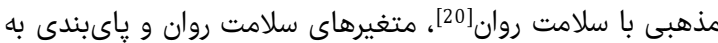

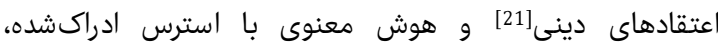

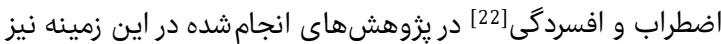
كزارش شده است بهعلاوه، مطالعات مربوط به ارتباط هوش درات معنوى، خشم و

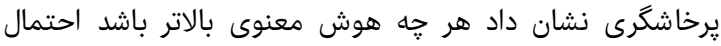

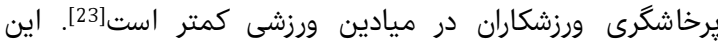

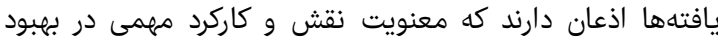

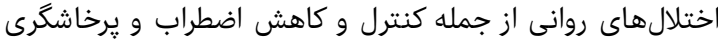

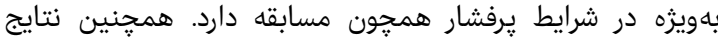

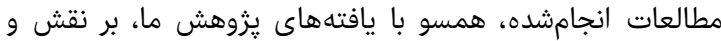


I\&V صفتى رقابتى يرخاشكرى رقابتى و خشم رقابتى بر اساس هوش معنوى ورزشكاران جانباز و معلول 6- Yang KP, Wu XJ. Spiritual intelligence of nurses in two Chinese social systems: A cross sectional comparison study. J Nurs Res. 2009;17(3):189-98.

7- Elkins M, Cavendish R. Developing a plan for pediatric spiritual care. Holist Nurs Pract. 2004;18(4):179-86. 8- Ghobari Bonab B, Motavalipoor A, Hakimirad E, Habibi Asgarabadi M. Relationship between anxiety and depression and magnitude of spirituality in students of Tehran. J Appl Psychol. 2009;3(2):110-23. [Persian]

9- Johnson SJ, Batey M, Holdsworth L. Personality and Health: The mediating role of trait emotional intelligence and work locus of control. Persnal Individ Differ. 2009;47(5):470-5.

10- Trevino KM, Pargament KI, Cotton S, Leonard AC, Hahn J, Caprini-Faigin CA, et al. Religious coping and physiological, psychological, social, and spiritual outcomes in patients with HIV/AIDS: Cross-sectional and longitudinal findings. AIDS Behav. 2010;14(2):379-89.

11- Joules Sh. The mediating role of God attachment between religiosity and spirituality and psychological adjustment in young adults. Columbus, Ohio: Ohio State University; 2007. pp. 3-41.

12- Beck R. Communion and complaint: Attachment, object-relations, and triangular love perspective on relationship with God. J Psychol Theol. 2006;34(1):4353.

13- Black JM, Hokanson Hawks J. Medical-surgical nursing: Management for positive outcomes. 8th edition. New York: Saunders; 2005.

14- Schmidt RA. Motor control and learning: A behavioral emphasis. Champaign, Ill.: Human Kinetics; 1941.

15- Karimi Y. Social psychology: Theories, concepts, and applications. 3rd edition. Tehran: Arasbaran Publication; 2008. [Persian]

16- Buss AH, Perry M. The aggression questionnaire. J Pers Soc Psychol. 1992;63(3):452-9.

17- Moalemi S, Bakhshani Noor M, Raghibi M. On the relationship between mental health, spiritual intelligence and dysfunctional attitudes in students of Systan and Baluchestan University, southeast of Iran. Q J Funam Ment Health. 2011;12(4);704-9. [Persian]

18- Moallemi S, Raghibi M, Salari Dargi Z. Comparison of spiritual intelligence and mental health in addicts and normal individuals. J Shahid Sadoghi Univ Med Sci. 2010;18(3):234-42. [Persian]

19- Dabiriyan P, Mahmoodi G, Vatankhah H. A comparison between intellectual aptitude and mental health in mothers having deaf, blind and normal children. J Except Educ. 2013;3(116):16-25. [Persian]

20- Ghahremani N, Nadi M. Relationship between religious / spiritual components, mental health and hope for the future in hospital staff of Shiraz Public Hospitals. Iran J Nurs. 2012;25(79):1-11. [Persian]

21- Azimirad G, Jalilvand M. Relationship between spiritual transcendence and competitive anxiety in male athletes. Int Res J Appl Basic Sci. 2012;3(9):1783-6. [Persian]

22- Bayrami M, Movahedi Y, Movahedi M. The role of spiritual intelligence in perceived stress, anxiety and depression of lorestan medical university students (Iran). J Babol UnivMed Sci. 2014;16(1):56-62. [Persian] 23- Behroozi H, Zare Zadeh M, Saberi Kakhki A. The relationship between spiritual intelligence and aggression among male teen soccer players. Sports Psychol Stud. 2013;6(4):81-94. [Persian]

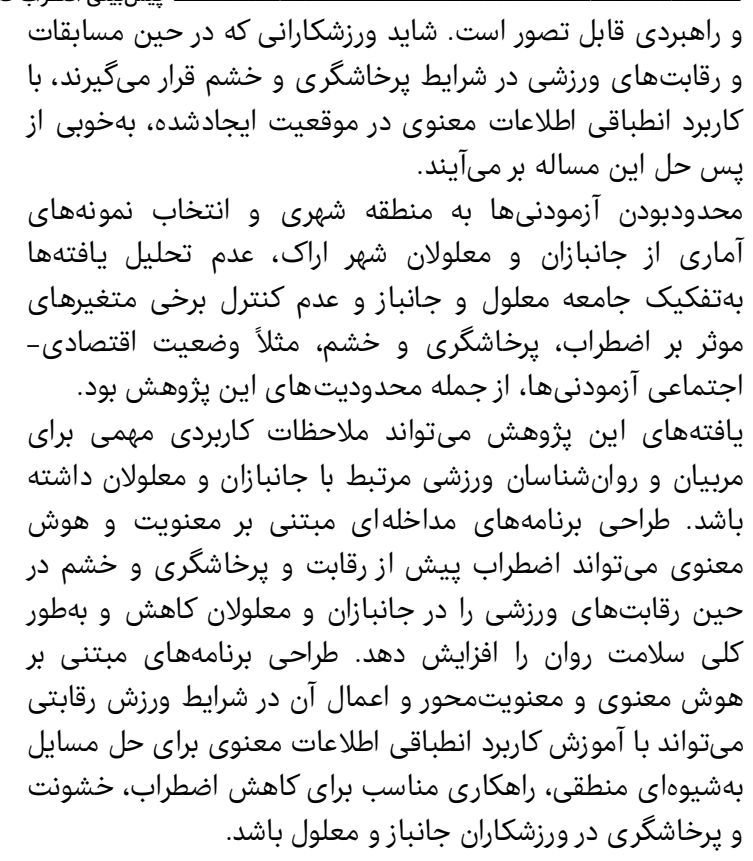

1- Totonchi M, Samani S, Zandi Ghashghaei K. Mediating role of self-concept in perfectionism and mental health of adolescents in the city of Shiraz in 2012. J Fasa Univ Med Sci. 2012;2 (3):210-7. [Persian]

2- Mehri A, Sedighy Some-Koochak Z. Assessment of mental health status and some related factors among students of Sabzevar Universities in 2010. Med Sci. 2012;21(4):293-304. [Persian]

3- Jenaabadi H, Nastiezaie N. A Comparison of aggression, anxiety, and depression in high school students in Saravan City. J Urmia Nurs Midwifery Fac. 2011;9(3):148-56. [Persian]

4- Murray RB, Zentner JP. Nursing concepts for health promotion. Nurs Educ Today. 1989;9(5):358.

5- Emmons A. Is spirituality intelligence? motivation cognition, and the psychology of ultimate concern. Int J Psychol Relig. 2000;10(1):3-26. 
women's national karate team. Res Sport Manag Motor Behav. 2016;5(10):27-35. [Persian]

31- Beygi A. Mohammadifar MA, Najafi M, Rezayi AM. Relationship of spirituality and forgiveness with family cohesion and adaptability in veterans. Iran J War Public Health. 2016;8(1):33-9. [Persian]

32- Haditabar H, Navabinejad S, Ahghar G. Effect of training spiritual intelligence on quality of life among veteran spouses. Iran J War Public Health. 2012;4(2):111. [Persian]

33- Pouraboli B, Hosseini SV, Miri S, Tirgari B, Arab M. Relationship between spiritual health and quality of life in post-traumatic stress disorder veterans. Iran J War Public Health. 2015;7(4):233-9. [Persian]

34- Nabatian E, Ghamarani A, Zakerian M, Mahdizadeh I. Relationship between spiritual health with quality of life veterans and disabled Birjand. Iran J War Public Health. 2013;5(2):35-9. [Persian]

35- Showani E, Zahrakar K, Ghasemi Jobaneh R, Dargahi Sh. Role of sexual satisfaction, spiritual health, psychological distress and self- esteem in marital commitment of veterans wives. Iran J War Public Health. 2015;7(2):99-105. [Persian]

36- Ja'fari Harafteh M, Ahmadi Mohammadabadi MR. A model for controlling aggressiveness, based on religious teachings. Ravanshenasi va Din. 2014;6(4):81-104. [Persian]
24- Delpisheh A, Mansourian M, Babakhani A, Bahami G, Bidel Z, Behzadifar M, et al. Province comparison of general health between veterans and their spouses in Ilam. Iran J War Public Health. 2013;6(1):27-33. [Persian]

25- Mahboobi M, Etemadi M, Khorasani E, Ghiasi M. The relationship between spiritual health and social anxiety in chemical veterans. Iran J Mil Med. 2012;14(3):186-91.

26- Khademvatani K, Aghakhani N, Esm-Hoseini G, Hazrati A, Alinezhad V, Nazari $\mathrm{H}$, et al . Study of relationship between spiritual health, anxiety and depression in acute myocardial infarction patients hospitalized in Seyyedoshohada Hospital in Urmia. Urmia Med J. 2015;25(12):1092-101. [Persian]

27- Haghshenas M, Noorbala A, Akabary SA, Negati Laeein V, Salehi M, Tayyebi Z. The study of relationship between spiritual intelligence and modes of students' attachment. Med Ethics J. 2011;14(4):167-81. [Persian]

28- Maxwell JP, Moores E. The development of a short scale measuring aggressiveness and anger in competitive athletes. Psychol Sport Exerc. 2007;8(2):179-93.

29- Fathi Rezaie Z, Abdoli B, Farsi A. Determination of validity and reliability of Farsi version of the competitive aggressiveness and anger scale among athletes. Sport Psychol Stud. 2014;7:1-14. [Persian]

30- Arbab B, Vaez Moosavi MK, Badami R. The relation between sports perfectionism and competitive anxiety in 